University of San Diego

Digital USD

Spring 5-26-2018

\title{
Power to the Patients: A Heart Failure Transitions of Care Program in a Heart Resource Center
}

Katherine Nicole Oxina Padiernos BSN, RN, DNP student

University of San Diego, kpadiernos@sandiego.edu

Scot Nolan DNP, RN, CNS, CCRN

nolan.scot@scrippshealth.org

Teri Armour -Burton PhD, CNML, NE-BC

teri.armour-burton@sharp.com

Colleen Austel Nadeau BSN, RN, CHFN

colleen.austel@sharp.com

Follow this and additional works at: https://digital.sandiego.edu/dnp

Part of the Nursing Commons

\section{Digital USD Citation}

Padiernos, Katherine Nicole Oxina BSN, RN, DNP student; Nolan, Scot DNP, RN, CNS, CCRN; Armour -Burton, Teri PhD, CNML, NE-BC; and Austel Nadeau, Colleen BSN, RN, CHFN, "Power to the Patients: A Heart Failure Transitions of Care Program in a Heart Resource Center" (2018). Doctor of Nursing Practice Final Manuscripts. 72.

https://digital.sandiego.edu/dnp/72

This Doctor of Nursing Practice Final Manuscript is brought to you for free and open access by the Theses and Dissertations at Digital USD. It has been accepted for inclusion in Doctor of Nursing Practice Final Manuscripts by an authorized administrator of Digital USD. For more information, please contact digital@sandiego.edu. 
Power to the Patients: A Heart Failure Transitions of Care Program in a Heart Resource Center Katherine Nicole O. Padiernos, BSN, RN, DNP-FNP Student; Scot Nolan, DNP, RN, CNS, CCRN; Teri Armour -Burton, PhD, CNML, NE-BC; Colleen Austel Nadeau, BSN, RN, CHFN University of San Diego

Author Note

Katherine Nicole O. Padiernos, BSN, RN, DNP-FNP Student at University of San Diego and Surgical Intensive Care Unit Registered Nurse at Sharp Grossmont Hospital.

This research was supported in part by resources from Sharp Grossmont Hospital.

Correspondence to this manuscript should be addressed to Katherine Nicole O. Padiernos, BSN, RN, DNP-FNP Student

Contact: kpadiernos@sandiego.edu 


\begin{abstract}
Background: In the United States an estimated 5.7 million adults have heart failure (HF), costing $\$ 30.7$ billion annually. National HF readmission rates have remained high at $21.3 \%$. After an extensive literature review, the purpose of this project was to incorporate teach back methods, self care education, resource accessibility, and increased post-discharge contact through an outpatient heart failure transitions of care program.
\end{abstract}

Methods and Results: Prior to discharge, HF patients were recruited and given a pre-test Self Care of Heart Failure Index (SCHFI). A DNP student and progressive care unit registered nurses provided discharge education utilizing a Healthy Heart Tracker booklet that focuses on medication adherence, symptom identification, low sodium diet, physical activity, and available resources. The patient was seen at the Heart Resource Center one-week post-discharge for a onehour education session. During weeks two through four the patient received one telephone follow-up call per week. The program finished with a one-hour HRC visit that provided a summation of education and post-test SCHFI. Pre and post values for both the SCHFI and HF readmission rates were utilized to evaluate the practice change. The total SCHFI score before and after the intervention showed an average 41-point improvement, achieving the project goal of $10 \%$ increase in each section. The study readmission rate was $7 \%$, achieving the $14.2 \%$ or less target goal and outperforming the hospital readmission rate of $15 \%$.

Conclusions: Outpatient HF programs is critical for increased provider and patient contact to allow for patient empowerment through self-care, barrier identification, and support team development. Standardized discharge with telephonic support may bridge the gap via coaching. Keywords: heart failure, readmissions, self care, transitions of care, teach back, telephone calls 
Power to the Patients: A Heart Failure Transitions of Care Program in a Heart Resource Center

\section{Introduction}

\section{Problem Description}

The American Heart Association (AHA; 2016) describes heart failure (HF) as a chronic condition that is characterized by inadequacy of the heart to pump sufficient amounts of blood throughout the body, which deprives the body of necessary amounts of oxygenation for cells to function properly. Negative outcomes may ensue due to HF such as acute exacerbations of HF that require readmissions due to compensation mechanisms of the heart to enlarge and retain fluid that may lead to severe fluid overload and shortness of breath (AHA, 2016). In the United States an estimated 5.7 million adults have heart failure, totaling a cost of $\$ 30.7$ billion every year for the nation (Centers for Disease Control and Prevention [CDC], 2016). National readmission rates related to $\mathrm{HF}$ have remained high at $21.3 \%$ as described in reports by the Centers for Medicare and Medicaid Services (CMS; 2014). Trends in data have shown that the prevalence of HF will increase to a rate of $46 \%$ by 2030 (AHA, 2016).

\section{Needs Assessment and Purpose}

The project facility is a large, urban hospital set in San Diego county that has a target goal of attaining the top decile benchmark of $14.2 \%$ or less readmission rate. Data collected over a twelve-month span of time provided by the project facility has shown an average readmission rate of $15 \%$ that falls outside of the target goal, outlining an opportunity for growth and practice change. The project facility has chosen and requested to direct initiatives at achieving the target goal for quality patient-centered care and value-based purchasing. Prior to the development of the project, the project facility did not have a transition of care heart failure program in a designated heart failure resource center. The project centers on the creation of a heart failure 
transitions of care program in a heart resource center (HRC) that includes standardized discharge teaching using teach-back methods, two one-hour nurse education visits post-discharge at a clinical site location within the facility, and telehealth in the form of telephone support calls. The purpose of the project was to keep 30-day readmission rates related to heart failure within the top decile benchmark as evidenced by improvement in patient self-care assessment values via the Self-Care of Heart Failure Index (SCHFI) screening tool, and a reduction of 30-day readmissions related to heart failure after a three-month intervention period.

\section{Framework}

The Iowa model was developed as a clinical decision making guide in the translation of evidence-based practice for clinicians and nurses through a flowchart that utilizes decision points for feedback loops (Melnyk \& Fineout-Overholt, 2015). It was initially developed in 1994 by the University of Iowa Hospitals and Clinics but has since then been updated to meet more current quality improvement requirements consistent with evidence-based practice (EBP) guidelines (Rycroft-Malone \& Bucknall, 2010). The Iowa model emphasizes on the organizational triggers and priorities, which have been the driving forces for this evidence-based practice initiative. The initial step includes identification of a problem-focused or knowledge-focused trigger, which stems from the organization's specific needs that align with the organization's top priorities. After doing so, formulation of a team occurs then review and synthesis of the literature. The key stakeholders including the chief nursing officer and project facility's heart and vascular team have emphasized their investment and support in this initiative, and have provided the necessary support team for the program to flourish. There is sufficient evidence accumulated from extensive literature review that support the program as safe and efficacious, and therefore may 
proceed with adoption of the clinical practice changes including continuous evaluation of the outcomes and dissemination of the results per the Iowa model.

\section{Methods}

\section{Participants and Recruitment}

Patients were recruited from one designated progressive care unit (PCU) at the project facility with the following inclusion criteria: HF as diagnosis either new diagnosis or acute on chronic, HF patients discharged from the one designated PCU in the facility consistent with the project timeline, able to provide consent to the program, able to perform self-care abilities, discharged to private residence, accessible via phone, able to access transportation, Englishspeaking, and able to read and write in English. Exclusion criteria included: recreational drug abuse listed under active problem list, physician/provider documentation, and/or screening test; uncontrolled psychiatric/mental health illness listed under active problem list, physician/provider documentation, and/or screening test; discharged to a skilled nursing facility or care facility other than a personal residence; unable to verbally communicate in fluent English; unable to perform self-care; very limited or complete limitation to phone access; and very limited or complete limitation to transportation.

The DNP student acting as the project coordinator tracked the HF patients for program eligibility on the "CHF Patient List" approved by the chief nursing officer provided by the clinical documentation specialist, which the DNP student has been granted access to by the HF senior specialist. The "CHF Patient List" encompasses information (e.g., patient name, facility unit location, age, medical record number, physician, insurance, admission from a skilled nursing facility, 30-day readmission classification) on all current HF patients coded with a HF diagnosis in order to ensure that all core quality measures are met prior to patient discharge. In order for 
the DNP student to stay updated on the discharge status of eligible HF patients, the PCU nurse manager provided the DNP student the "Projected/Confirmed Discharge Tracking List" and “Throughput Discharge Tracking Form" via daily email Monday through Friday. Having the list of all HF patients allowed the DNP student to perform chart review of all patients to deem eligibility via the inclusion and exclusion criteria mentioned previously. The DNP student performed chart review and recruited patients approximately three days a week, depending on the eligibility of the patients and availability of the DNP student to see the patient within the outlined timeline. Prior to initiation of the program interventions and processes, the Institutional Review Boards of the project facility and University of San Diego granted exemption to the DNP project as it did not classify as research or clinical investigation per federal definition criteria. Both Institutional Review Boards gave clearance for the DNP student to obtain informed consent of eligible patients through an initial face-to-face encounter at PCU bedside prior to discharge.

\section{Project Interventions}

Prior to discharge, recruitment according to the above mentioned processes occurred at the patient's bedside by the DNP student. The DNP student provided and educated the participant with a Healthy Heart Tracker booklet that focuses on medication adherence, symptom identification, low sodium diet, physical activity, and available resources. At the end of educating the patient on the provided booklet, the DNP student assessed the patient's knowledge with a pop-quiz created by the DNP student based on the information from the booklet. The popquiz employs teach-back with five key points, which allowed the DNP student to identify education gaps and provide positive re-enforcement for knowledge retained by the participant. The DNP student also provided education to the PCU nurses on ensuring each HF patient received education on the booklet, as well as engaging the patient with the pop-quiz prior to 
discharge to allow for teach back methods by the PCU nurse prior to discharge. Increased teachback moments allow further opportunities to solidify the information provided by the DNP student, and also gauge the patient's retention of knowledge and self-care capabilities (Ha Dinh, Bonner, Clark, Ramsbotham, and Hines, 2016). Self-care accountability was placed into action by the DNP student through educating and enrolling them in the program. The program incorporated self-care behaviors including medication adherence, identification and monitoring of symptoms such as weight gain using a weight log provided prior to hospital discharge, sustaining a low sodium diet, and integrating a lifestyle of physical activity (Dickson, Buck, \& Riegel, 2011).

Accessibility of resources and increased post-discharge point of contact increases patient self-care and decreases the risk for readmissions (Leppin et al., 2014). An appointment for the patient was made at the HF Resource Center for one-week post discharge for the one-hour education session. Appointments to all follow-ups were made and agreed upon between the DNP student and participant to increase accountability. Each participant was provided with a personalized schedule for specific dates and times for each follow-up appointment consistent with the project's timeline as follows: one-hour education session using teach back principles and booklet week one post-discharge; one telephone support call per week to re-enforce education with booklet, assess self-care, address barriers, and provide education and emotional support during weeks 2 through 4 post-discharge; and a final one hour education session and support visit using booklet, and administration of the post-test SCHFI during week five postdischarge.

Prior to discharge during the recruitment phase, the SCHFI version 7.1 was provided to the patient to assess their baseline knowledge and obtain self-care values related to $\mathrm{HF}$ 
maintenance, monitoring, management, and confidence (Liou et al., 2015). Emphasis on selfcare education and assessment of these values using validated tools is proven to be effective by Clark et al. (2014). The SCHFI version 7.1 is a newly revised tool with reliability testing underway; however, the project facility's heart and vascular team requested this version be used in the facilitation of this HF transitions of care program as it is currently being administrated for another initiative with clearance previously obtained by the project facility's Institutional Review Board (IRB). The DNP student also received permission to use the SCHFI version 7.1 and proceed with this new tool, with approval given by the DNP student's university faculty advisor and the creator of the tool in an email inquiry. Previous versions have received global reliability index coefficients for each subscale with values sufficient for multidimensional scales, e.g. a coefficient alpha of 0.827 for SCHFI version 6 (Riegel, Lee, Dickson, \& Carlson, 2009).

At the first education session during week one post-discharge, the DNP student reenforced the project facility's "Healthy Heart Tracker" booklet developed by the project facility's heart failure team. This workbook allows the patient to engage and become an active participant in their health and clarify any questions with the teach back method. Further reinforcement of self-care empowerment and teaching was employed through twenty-to-thirtyminute telephone support calls by the DNP student once a week for weeks two through four postdischarge, which again reviewed the maintenance of aforementioned self-care behaviors as well as the identification of barriers to optimal self-care management. A standardized telephone follow-up form based on the booklet developed by the DNP student approved by the project facility was used to further re-enforce the education being provided. The final visit on the fifth week post-discharge was a one-hour education session, which focused on any educational gaps the patient had, barriers the patient identified, and a post-test SCHFI to compare the significance 
of the HF transitions of care program. Reinforcement and increased patient education encounters for added self-care management support has been further identified as an effective evidencebased practice initiative by Baker et al. (2011). The combination of providing patients with written education materials and reviewing it with them and providing frequent monitoring and counseling via telephone calls was established by Liao et al. (2015) as beneficial with significant increases in self-care maintenance, monitoring, management, and confidence utilizing a pre and post SCHFI.

\section{Measures}

The outcome indicators for this project are the pre and pos- SCHFI assessment values and readmission rates. Patients involved in the project were given a pre-test SCHFI to complete prior to the initial education session at the HRC, and subsequently given a post-test SCHFI to complete at the final education session and support visit at the HRC during the last week of the transition program. This allowed for comparison of baseline knowledge to knowledge acquired from the transition program by assessing HF maintenance, monitoring, management, and confidence (Liou et al., 2015). Completion of the forms was monitored weekly to ensure that each patient did not fall outside of the time sensitive completion dates to ensure accuracy of the values pre and post evidence-based practice changes. Due to the increased accountability of selfcare placed onto the patients by the DNP student through education and enrollment into the transitions of care program, patient self-care behaviors were anticipated to positively increase throughout the program and be evident at the completion (Dickson, Buck, \& Riegel, 2011). There was an anticipated increase of each self-care assessment value by $10 \%$ in each section of the SCHFI. 
The second outcome indicator for this project is the monitoring and analysis of the heart failure readmission rates. The project facility requested initiatives to achieve the top decile benchmark of less than $14.2 \%$. Thus, the goal for this project was to provide an initiative for the project facility to obtain this goal and with implementation of this project to showcase a decreased heart failure readmission rate that meets the target goal of less than $14.2 \%$. The project compared the hospital's baseline readmission rates related to heart failure for a 12-month span during pre-intervention, followed by three months of collection during post-intervention. This allowed comparison of pre and post values to provide accurate depiction of impact. The collection of this outcome indicator is continuously monitored by the hospital, and was given permission of access for data retrieval for the DNP project purposes.

\section{Data Analysis}

Analysis of pre and post SCHFI assessment values was completed for all four sections of the self-care management tool. This allows the DNP student, stakeholders, and future audiences to compare the difference and see the impact increased education through a transitions program for heart failure patients can make. Measuring self-care values and subsequently anticipating seeing a rise in these values showcases the empowerment of patients to safely transition from inhospital to outpatient to prevent negative sequelae. Secondly, readmission rates related to HF was collected and analyzed for comparison pre and post DNP project implementation. To gather an accurate depiction of impact, baseline values from the clinical site was gathered over a twelve-month span with post-intervention data starting from project implementation date of October 2017 to the end date of December 2018. The readmission rates were calculated by assessing percentages through utilization of the number of heart failure readmission as the numerator with the total number of heart failure patients as the denominator for each individual 
month. This allows for reduction of fluctuation of values regardless of the varying amount of patients per month. With the increase in patient education and advocating self-care principles, there was a reflection of a decreased readmission rate.

The data collected by the DNP student was de-identified at the project site, and destroyed in an identified protected health information bin recognized by the project facility after completion of the project. Data collection forms contained a section at the top of the form for patient labels containing identifying information (i.e., 001, 002, 003, etc.). Data was saved electronically on an approved USB drive provided by the project site. After entering the data in Excel, forms were shredded by the performance improvement team. Appropriate data power analyses methods were utilized including bar charts, control charts, and pareto charts as deemed appropriate per the university statistician collaborator.

\section{Results}

Initially the project started with eight participants that met the inclusion criteria and did not meet the exclusion criteria, and were willing to participate in the transitions of care program. However, one participant was readmitted before meeting the one-week post-discharge mark and before meeting the DNP student for the first follow-up visit. That participant, however, had a poor prognosis and subsequently was placed into hospice. A second participant from the pilot group of eight participants was readmitted prior to the second telephone follow-up call. That particular participant completed the first and second follow-up appointments, but was unable to be contacted via phone number thereafter. Despite multiple attempts by the DNP student to call the participant, the DNP student was met by unanswered voicemails or family members that stated the patient was unavailable. 
Participant sociodemographic information was collected for all eight participants in order to compare varying characteristics among the individuals. The age span of the participants ranged from 40 to 94 years old, with an average of an age of 72 years old. There were three male participants and five female participants. Three ethnicities were represented in the pilot group: three Asian, two Black/African-American, and three White/Caucasian. The project facility's heart failure team was highly interested in the psychosocial element when assessing participants' success outcome. Of the eight participants, four were married, one divorced, one widowed, and two single with only one participant living alone. However, all participants stated they had a support system in place. These varying psychosocial elements did not correlate with completion or pre- and post- values of the program, as the patients that did not complete the study were both married, did not live alone, and had a support system. Participants' education level was collected, showing three participants had less than high school education, three participants had high school completion, one with an Associate's Degree, and one Bachelor's Degree. Seven participants were retired while one participant was still working. When questioned about health perception, $25 \%$ of the participants responded with "good" and $75 \%$ responded with "fair." Diagnosis duration was calculated, displaying $75 \%$ of participants had the diagnosis for less than or equal to five years, $12.5 \%$ of participants had the diagnosis for six to ten years, and $12.5 \%$ of patients had the diagnosis for greater than ten years. All participants identified as having at least one or more comorbidities.

The project's average readmission rate over a three-month span was $7 \%$, achieving the $14.2 \%$ or less target goal and outperforming the hospital's average readmission rate over a twelve-month span of $15 \%$ that did not meet the target goal. This information is seen below in Figure 1 displayed on a control chart that plots each individual readmission rate that accounts for 
the different denominators with the varying total amount of HF patients per month, and applies statistics to obtain the average rate for the hospital's readmission rate without the practice change and the project's readmission rate with the practice change.

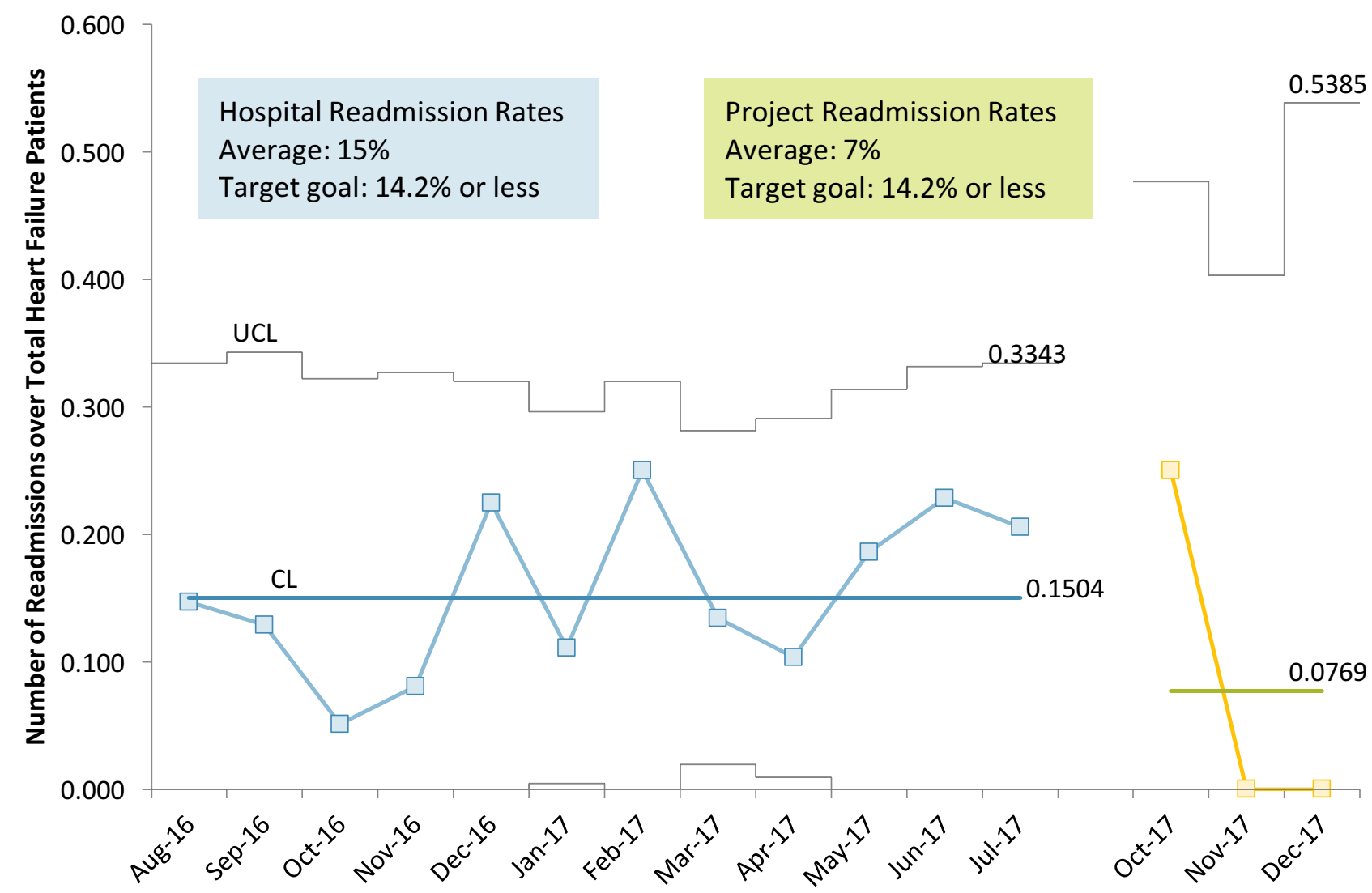

Figure 1. Comparison of hospital status quo to project intervention of average readmission rates for heart failure patients.

Calculating the total SCHFI score before project intervention yielded 136 points and the total SCHFI score after project intervention was 177 points, thereby displaying a 41-point improvement from baseline and achieving the project goal of $10 \%$ increase in each section of SCHFI. All six participants on an individual level that completed the program had an increase of SCHFI score baseline in each section after the program. This can be seen in the bar chart below 
in Figure 2. When reviewing each SCHFI category, it is beneficial for patients and stakeholders to analyze which section(s) had the greatest impact. This can be seen in Figure 3 displayed below in a pareto chart that arranges each category by frequency. The section monitoring alone accounted for 39\% of all improvements indicating the greatest impact. For combined monitoring and confidence sections, the two sections accounted for $68.2 \%$ of all improvements. This allows providers and stakeholders to assess which categories the patients thrived in and found the most value for growth opportunities. Anecdotally, the participants gave feedback to the DNP student that previous to the program, they did not have the monitoring or evaluation knowledge and tools, and therefore did not feel confident in self-care capabilities. This information correlates with the data collected below of significant increases in the two areas the patients felt the weakest, and allowed for growth and improvements.

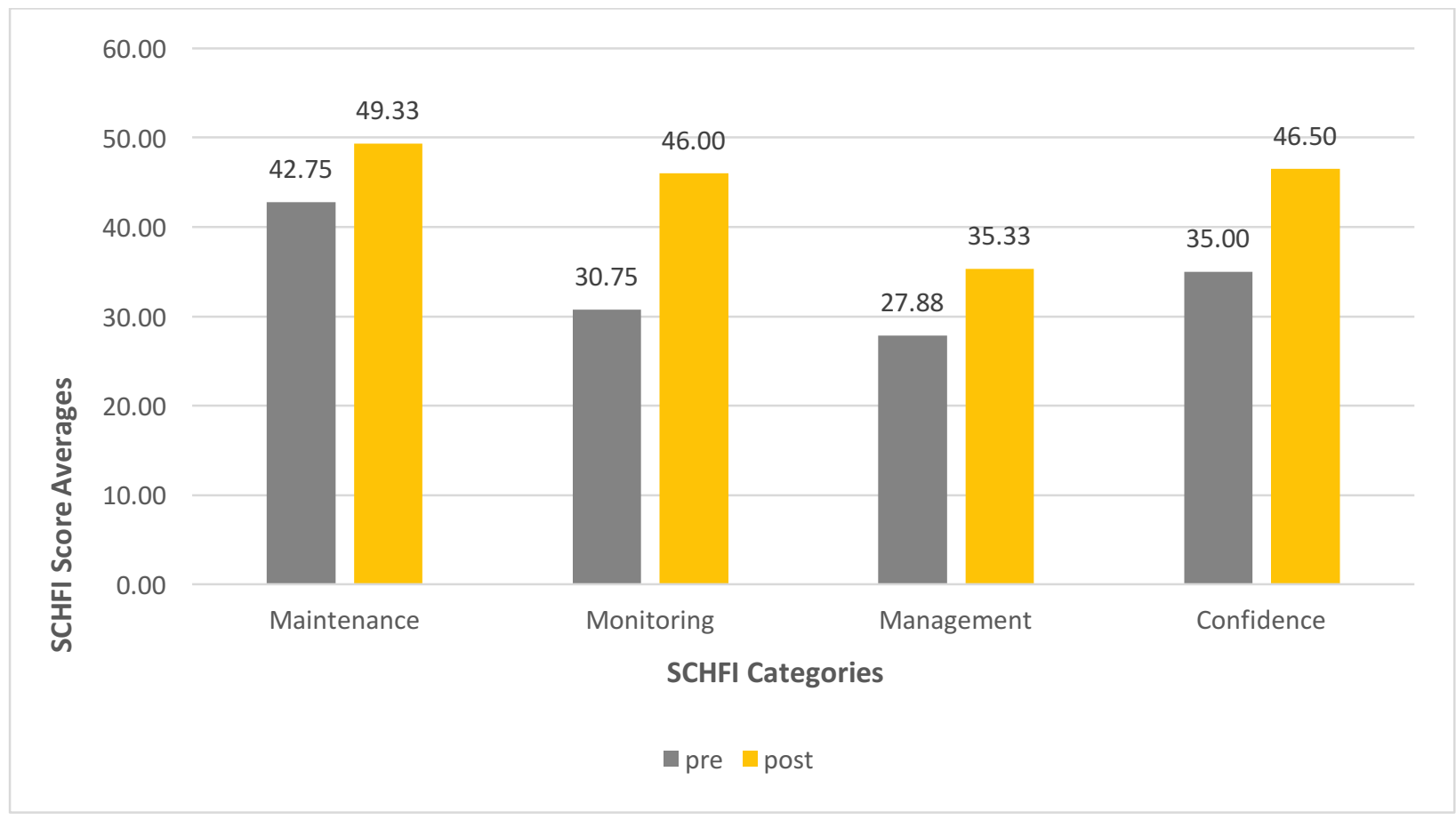

Figure 2. Self Care of Heart Failure Index category averages before and after project intervention. 


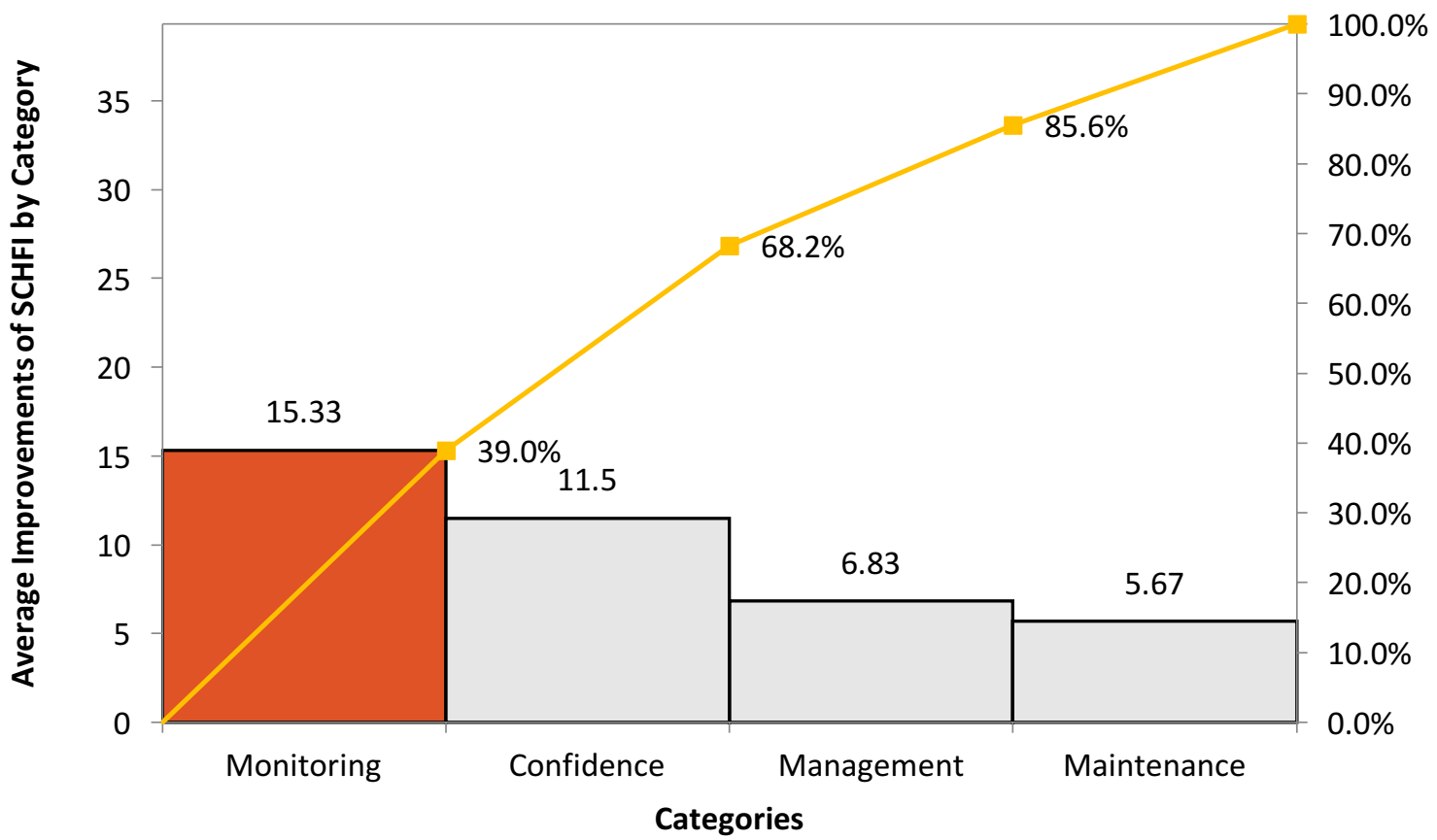

Figure 3. Self Care of Heart Failure Index average improvements by category.

\section{Discussion}

\section{Cost/Benefit Analysis}

Implementation of a heart failure transitions of care program is a cost-effective method focused on value-based purchasing principles and patient-centered care. Return on investment will be realized through cost savings from prevention of penalties from the Hospital Readmissions Reduction Program and reduced readmission costs with cost avoidance. Based on a study by Gardner et al. (2014) that reviewed the cost effects of care transitions interventions, increased and standardized discharge teaching interventions significantly lowered hospital utilization and cost avoidance per patient was approximately $\$ 3,752$ to reflect Medicare payments. With the anticipated HF readmission rates reduction of less than $14.2 \%$, there will be a subsequent reduction toward readmission fees. Cost avoidance would also be realized through the prevention of fees related to complications, both anticipated and unanticipated, with heart 
failure readmissions that is not depicted in this cost-benefit analysis. A detailed description of the cost-benefit analysis of a heart failure transitions of care program is displayed below in Table 1.

Table 1

\begin{tabular}{|c|c|}
\hline \multicolumn{2}{|c|}{$\begin{array}{l}\text { Cost-Benefit Analysis of a Heart Failure Transitions of Care Program in a Heart Resource } \\
\text { Center }\end{array}$} \\
\hline \multicolumn{2}{|l|}{ Costs } \\
\hline Description & Total \\
\hline $\begin{array}{l}1 \text { Advanced Practice Registered Nurse } \\
\text { (APRN) }\end{array}$ & 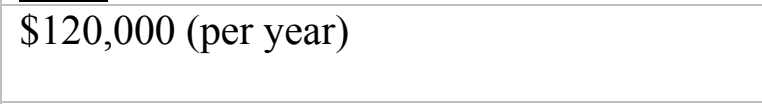 \\
\hline 4 East PCU RN training & $\begin{array}{l}\$ 0 \text { : Incorporated within meeting and in- } \\
\text { service training }\end{array}$ \\
\hline 4 East PCU Charge and Lead RN training & $\begin{array}{l}\$ 0 \text { : Incorporated within pre-established } \\
\text { meeting }\end{array}$ \\
\hline Self-Care of Heart Failure Index tool & $\begin{array}{l}\$ 0 \text { : No additional cost, already provided by } \\
\text { project facility with IRB approval }\end{array}$ \\
\hline SGH Heart Healthy Tracker & $\begin{array}{l}\$ 0: \text { No additional cost, already provided by } \\
\text { project facility }\end{array}$ \\
\hline Miscellaneous education materials & $\begin{array}{l}\text { \$0: No additional cost, already provided by } \\
\text { project facility }\end{array}$ \\
\hline Total Costs: & $\$ 120,000$ \\
\hline \multicolumn{2}{|l|}{ Benefits through Cost Avoidance } \\
\hline Description & Per Year \\
\hline Heart failure readmission & $\begin{array}{l}\$ 15,667^{1} \times 64^{2}=\$ 1,002,688 \\
\$ 1,002,688 \times 0.15(\% \text { decrease after } \\
\text { intervention })=\$ 150,403.20\end{array}$ \\
\hline Cost-benefit analysis: & $\begin{array}{l}\$ 150,403.20 / \$ 120,000=1.25 \\
\text { For every dollar spent in the program, } \$ 1.25 \\
\text { in benefit was achieved }\end{array}$ \\
\hline Expected cost savings: & $\$ 124,800-\$ 120,000=\$ 30,403.20$ \\
\hline
\end{tabular}

\section{Limitations}

The sample size of the project was small with a total of eight participants enrolled and six participants that completed. Although this project served as a pilot for the transitions of care program and accounted for the small sample size by using denominators to produce averages to account for varying sizes, the project may not have captured a true reflection of the practice 
change if there were a larger number of participants. Due to the exclusion criteria made in collaboration with the project facility heart and vascular team, many patients screened within the project timeline were deemed ineligible to participate. This may be contributed to the particular section of the county's population group. In addition, clearance by the project facility was given to the DNP student to recruit from a total of two PCUs. However, due to a research study being conducted in the project facility, only one of the PCUs remained available for project inclusion. The short duration of three months for the practice change may not have been sufficient to correlate the positive results to the practice change. However, the positive results showcase a promising initiative and a longer implementation duration is therefore warranted. The patient that was unable to complete the program due to severity of heart failure should have been captured in screening and possibly referred to hospice earlier per health care provider discretion. The patient lost to follow-up that subsequently resulted in a readmission despite multiple attempts to reconnect by the health care team may yield a loss in productivity hours for the health care team.

\section{Conclusions and Implications for Practice}

It is anticipated that the issue of hospital readmissions related to HF will lead to continued deleterious sequelae unless further action is taken to achieve and maintain the set goals by CMS. Patient-centered care should be focused on safety initiatives, which include providing the patients with accessible resources for successful transition from the inpatient to outpatient environment. Standardized discharge teaching that utilizes teach back method, assessing pre and post self-care assessment values, follow-up education session within one week of hospital discharge utilizing an interactive workbook, and frequent telephone support calls at specific intervals, will provide patients the appropriate tools and lessons to move forward. Positioning registered nurses and advanced practice registered nurses at the forefront of these initiatives is a 
critical key in helping piece together the equation to achieve decreased readmission rates and increased positive patient outcomes as evidenced by increased self-care assessment values and decreased readmission rates related to $\mathrm{HF}$. 


\section{References}

American Heart Association (2016). About heart failure. Retrieved from http://www.heart.org/HEARTORG/Conditions/HeartFailure/AboutHeartFailure/What-isHeart-Failure_UCM_002044_Article.jsp\#.WNK8ORBVGGQ

Baker, D. W., Dewalt, D. A., Schillinger, D., Hawk, V., Ruo, B., Bibbins-Domingo, K., .. . Pignone, M. (2011). The effect of progressive, reinforcing telephone education and counseling versus brief educational intervention on knowledge, self-care behaviors and heart failure symptoms. Journal of Cardiac Failure, 17(10), 789-796. doi:10.1016/j.cardfail.2011.06.374

Centers for Disease Control and Prevention (2016). Heart failure fact sheet. Retrieved from https://www.cdc.gov/dhdsp/data_statistics/fact_sheets/fs_heart_failure.htm

Centers for Medicare and Medicaid Services (2014). Medicare hospital quality chartbook 2014.

Retrieved from https://www.cms.gov/medicare/quality-initiatives-patient-assessmentinstruments/hospitalqualityinits/downloads/medicare-hospital-quality-chartbook2014.pdf

Centers for Medicare and Medicaid Services (2016). Hospital compare datasets. Retrieved from https://data.medicare.gov/data/hospital-compare

Clark, A. P., McDougall, G., Riegel, B., Joiner-Rogers, G., Innerarity, S., Meraviglia, M., . . . Davila, A. (2015). Health status and self-care outcomes after an education-support intervention for people with chronic heart failure. Journal of Cardiovascular Nursing, 30(4 Suppl 1), S3-13. doi:10.1097/jcn.0000000000000169 
Dickson, V. V., Buck, H., \& Riegel, B. (2011). A qualitative meta-analysis of heart failure selfcare practices among individuals with multiple comorbid conditions. Journal of Cardiovascular Failure, 17(5), 413-419. doi:10.1016/j.cardfail.2010.11.011

Ha Dinh, T. T., Bonner, A., Clark, R., Ramsbotham, J., \& Hines, S. (2016). The effectiveness of the teach-back method on adherence and self-management in health education for people with chronic disease: a systematic review. JBI Database Systematic Reviews And Implementation Reports, 14(1), 210-247. doi:10.11124/

Leppin, A. L., Gionfriddo, M. R., Kessler, M., Brito, J. P., Mair, F. S., Gallacher, K., . . . Montori, V. M. (2014). Preventing 30-day hospital readmissions: a systematic review and meta-analysis of randomized trials. JAMA Internal Medicine, 174(7), 1095-1107. doi:10.1001/jamainternmed.2014.1608

Liou, H. L., Chen, H. I., Hsu, S. C., Lee, S. C., Chang, C. J., \& Wu, M. J. (2015). The effects of a self-care program on patients with heart failure. Journal of the Chinese Medical Association, 78(11), 648-656. doi:10.1016/j.jcma.2015.06.004

Riegel, B., Lee, C. S., Dickson, V. V., \& Carlson, B. (2009). An update on the self-care of heart failure index. Journal of Cardiovascular Nursing, 24(6), 485-497. doi: 10.1097/JCN.0b013e3181b4baa0 